\title{
Platelet markers correlate with glycemic indices in diabetic, but not diabetic-myelodysplastic patients with normal platelet count
}

\author{
Maria Dalamaga ${ }^{\mathrm{a}, *}$, Konstantinos Karmaniolas $^{\mathrm{b}}$, Antigoni Lekka ${ }^{\mathrm{b}}$, George Antonakos ${ }^{\mathrm{a}}$, \\ Apollon Thrasyvoulides ${ }^{\mathrm{a}}$, Evangelia Papadavid ${ }^{\mathrm{c}}$, Nikolaos Spanos $^{\mathrm{a}}$ and Amalia Dionyssiou-Asteriou ${ }^{\mathrm{a}}$ \\ a Department of Clinical Biochemistry, Medical School, University of Athens, "Attikon” General University \\ Hospital, Athens, Greece \\ ${ }^{\mathrm{b}}$ Department of Hematology Laboratory, NIMTS General Hospital, Athens, Greece \\ ${ }^{\mathrm{c}}$ Department of Internal Medicine-Dermatology Section, Medical School, University of Athens, “Attikon” General \\ University Hospital, Athens, Greece
}

\begin{abstract}
Background: Altered thrombocyte morphology and function have been reported in patients with diabetes mellitus (DM) type 2. The aim of the present study was to determine the associations between platelet morphology markers and hemoglobin $\mathrm{A} 1 \mathrm{C}\left(\mathrm{HbA}_{1 c}\right)$, fasting glucose $(\mathrm{FG})$, hypertension and coronary heart disease (CHD) in patients with myelodysplastic syndromes (MDS) and DM, in patients with DM and in controls.

Methods: This cross-sectional study included 30 cases with primary MDS with normal platelet count and non-insulin dependent diabetes, 30 non-insulin dependent diabetic patients and 30 non-diabetic, non-MDS controls matched on age and gender.

Results: After adjusting for body mass index, platelet number, $\mathrm{CHD}$ and hypertension, $\mathrm{HbA}_{1 c}$ and $\mathrm{FG}$ were significant predictors of mean platelet volume (MPV) and platelet distribution width (PDW) in diabetic patients. There was no correlation between platelet parameters and $\mathrm{HbA}_{1 c}$ or $\mathrm{FG}$ in diabetic MDS patients. In controls, FG and hypertension predicted significant differences in platelet morphology. Platelet count correlated with platelet morphology in diabetic MDS and control groups, but not in diabetics.

Conclusions: MPV and PDW are associated with glycemic indices in diabetic patients but not in diabetic MDS patients with normal platelet counts. Non-diabetic controls also exhibit FG related changes in platelet morphology. This suggests other factors inherent to bone marrow dysplasia, platelet turnover and biochemistry, or vascular environment affect platelet morphology in diabetic MDS patients even with normal platelet count. Platelet morphology in this population may be an early marker for myelodysplasia. These findings also support platelet morphology change as a marker for elevated macrovascular disease risk.
\end{abstract}

Keywords: Myelodysplastic syndrome, diabetes, glycohemoglobin, glucose, mean platelet volume

\footnotetext{
*Corresponding author: Maria Dalamaga, MD, MS, MPH, PhD, Ass. Professor in Clinical Biochemistry, 19, 28th October, Agia Paraskevi, Post Code 15341 Athens, Greece. Tel.: +30 6977440344; Fax: +30 2106082467; E-mail: madalamaga@med.uoa.gr.
}

\section{Introduction}

Altered thrombocyte morphology and function have been reported in patients with diabetes mellitus type 2 [1]. Mean platelet volume (MPV) as well as platelet distribution width (PDW) have been evaluated as markers of platelet morphology and activation in many stud- 
ies [1-3]. There is accumulating evidence that MPV reflects the state of thrombogenesis [4]. Diabetic patients present higher MPV values than normal controls matched on age and gender [1,5]. High MPV values are related with an increased risk of developing microand macrovascular diabetic complications such as cardiovascular disease $[1,6,7]$. Moreover, it has recently been reported that MPV is lower in thrombocytopenic patients with bone marrow disease and could act as a surrogate marker for the degree of bone marrow disease [8] in patients with myelodysplastic syndrome (MDS). However, the relationship of platelet parameters to the development of diabetic vascular disease and especially in MDS patients with diabetes mellitus (DM) and normal platelet count remains unclear. To the best of our knowledge, this is the first study in medical literature assessing the association of glycosylated hemoglobin and fasting blood glucose with platelet parameters in diabetic MDS patients with normal platelet count.

Therefore, the aim of the present investigation was (1) to evaluate platelet markers such as MPV and PDW in diabetic MDS patients with normal platelet count, in patients with DM type 2 without microvascular diabetic complications, and in non-diabetic non-MDS healthy controls; and (2) to determine the associations between platelet parameters (MPV, PDW) and glucosylated hemoglobin $\left(\mathrm{HbA}_{1 c}\right)$, fasting blood glucose, age, gender, body mass index (BMI), hypertension and coronary heart disease (CHD) in these patients.

\section{Materials and methods}

In this study, both cases and controls were selected from patients of the Outpatient Department at the Veterans' General Hospital of Athens (NIMTS). This hospital is the only Veterans' Hospital in Athens Metropoli$\tan$ area and the entire Southern Greece. The study included 90 patients divided into three groups. Group A comprised 30 cases with primary MDS and type 2 diabetes mellitus (21 men and 9 women) with a mean age of $72.6 \pm 6.1$, a mean duration of diabetes of $10.7 \pm$ 4.2 years and a mean platelet count of $177.9 \times 10^{9} / \mathrm{L} \pm$ 28.5 (range: $150-290 \times 10^{9} / \mathrm{L}$ ). Eligible cases of this group included newly diagnosed patients with histologically confirmed primary MDS without underlying malignancy, prior to any therapeutic approach (transfusion and/or chemotherapy) under age 82 , consecutively seen in the Outpatient Department of Internal MedicineHematology between March 1, 2000 and October 31,
2006. All cases were classified according to the FAB Cooperative Group scheme [9]. The following MDS subtypes were recognised: refractory anaemia (RA), refractory anaemia with ring sideroblasts (RARS) and refractory anaemia with excess blasts (RAEB).

Group B consisted of 30 patients with type 2 diabetes mellitus (21 men and 9 women) with a mean age of $70.2 \pm 6.1$ and a mean duration of diabetes of $13.2 \pm$ 3.6 years. These were patients without neoplastic and infectious conditions from the Outpatient Diabetologic Department of the same hospital matched to cases of group A on age ( \pm 5 years) and gender.

Group C (healthy controls) included 30 non-diabetic individuals (21 men and 9 women) with a mean age of $73.5 \pm 5.9$ without neoplastic and infectious conditions seen in the Outpatient Laboratory Department from individuals coming for an annual check-up examination of the same hospital and matched to cases of group A on age ( \pm 5 years) and gender. No control developed MDS or any malignancy. For every eligible case of group A, an attempt was made to randomly identify a case of group B and a control of group C admitted to the Outpatient Department of Veterans' Hospital as closely as possible in time to the corresponding case.

Exclusion criteria for all participants in the study were detection of abnormal platelet count (less than $150 \times 10^{9} / \mathrm{L}$ ), abnormal leucocyte count (greater than $10 \times 10^{9} / \mathrm{L}$ ), presence of iron deficiency anemia or hemolytic anemia. None of the patients and controls had any thrombotic disease or had received anticoagulant or antiplatelet medication. Furthermore, all patients were not smokers (or were ex-smokers for at least 2 years) and didn't present hypertriglyceridemia (serum triglycerides greater than $180 \mathrm{mg} / \mathrm{dL}$ ) or hyperbilirubinemia (serum bilirubin greater than $1.5 \mathrm{mg} / \mathrm{dL}$ ). All study participants were fully informed of the aim of the study and gave written consent for their participation and their agreement that the results of this study may well be presented or published, solely in the interests of science, provided that their anonymity is maintained. The study protocol was approved by the Committee of NIMTS Hospital.

Platelet count, MPV and PDW were measured in blood samples anticoagulated with sodium citrate within 90 min after collection by venipuncture. Each measurement was performed in two different automated blood cell counters (Sysmex XE 2100 and Cell Dyn 1700). During the study period, the anticoagulant, its dilution, the blood cell counters as well as the time from venipuncture and the conditions of sample storage remained invariable. $\mathrm{HbA}_{1 c}$ was measured using 
Table 1

Demographic and clinical data of diabetic MDS patients (Group A), diabetic patients (Group B) and healthy controls (Group C)

\begin{tabular}{lcccc}
\hline Parameters & Group A & Group B & Group C & p value \\
\hline Number of patients & 30 & 30 & 30 & - \\
Age, years, mean $\left(\mathrm{SD}^{\partial}\right)$ & $72.6(6.1)$ & $70.2(6.1)$ & $73.5(5.9)$ & $\mathrm{NS}^{\dagger}$ \\
Gender (male/female) & $21 / 9$ & $21 / 9$ & $21 / 9$ & $\mathrm{NS}$ \\
Duration of diabetes, years, mean (SD) & $10.7(4.2)$ & $13.2(3.6)$ & - & 0.02 \\
Fasting blood glucose, mmol/L, mean (SD) & $7.8(0.9)$ & $8.5(0.9)$ & $5.1(0.5)$ & $<0.001$ \\
$\mathrm{HbA}_{1 c}, \%$, mean $(\mathrm{SD})$ & $7.1(1.1)$ & $8.1(0.8)$ & $5.2(0.4)$ & $<0.001$ \\
Mean platelet count, $\times 10^{9} / \mathrm{L}$, mean (SD) & $177.9(28.5)$ & $261.2(36.1)$ & $268.9(47.6)$ & $<0.001$ \\
MPV, fL, mean (SD) & $9.5(1.2)$ & $13(0.9)$ & $11.2(1.2)$ & $<0.001$ \\
PDW, mean $(\mathrm{SD})$ & $13.1(0.9)$ & $16.4(1.3)$ & $13(1.7)$ & $<0.001$ \\
Body Mass Index, kg/m ${ }^{2}$, mean (SD) & $26(0.9)$ & $27.8(1.4)$ & $26.4(1.1)$ & $<0.001$ \\
Arterial hypertension $($ yes/no) & $9 / 21$ & $8 / 22$ & $4 / 26$ & $\mathrm{NS}$ \\
Treatment for hypertension (yes/no) & $2 / 28$ & $4 / 26$ & $1 / 29$ & $\mathrm{NS}$ \\
Coronary artery disease (yes/no) & $4 / 26$ & $4 / 26$ & $1 / 29$ & $\mathrm{NS}$ \\
FAB MDS category $(\mathrm{N}$ of patients and $\%)$ & & & & - \\
RA & $17(56.7)$ & - & - & - \\
RARS & $10(33.3)$ & - & - & - \\
RAEB & $3(10)$ & - & & - \\
\hline
\end{tabular}

${ }^{\dagger} \mathrm{NS}=$ Non-significant

${ }^{\partial} \mathrm{SD}=$ Standard Deviation.

high-performance liquid chromatography (G7 TOSOH Glycohemoglobin analyzer HLC-723, TOSOH Corporation, Japan). Fasting blood glucose was determined by glucose oxidase method. In groups $\mathrm{A}$ and $\mathrm{B}$, diabetes was diagnosed according to the American Diabetes Association criteria [10]. The laboratory exams performed at the time MDS was diagnosed were bone marrow and trephine biopsy supplemented by cytogenetics. Complete laboratory investigation, detection of microalbuminuria (immunochemical method), calculation of body mass index based on weight and height $\left(\mathrm{BMI}=\right.$ weight $/$ height $\left.^{2}\right)$ and funduscopy were performed in all patients. Weight and height measurements were performed by the same resident in the hospital under strictly similar conditions at the same time in the morning. Diagnosis of coronary artery disease was based upon positive stress testing and/or history of myocardial infarction (Minessota coding). Finally, hypertension was defined as detection on three separate occasions of systolic pressure higher than $140 \mathrm{mmHg}$ and/or diastolic pressure higher than $90 \mathrm{mmHg}$ as well as use of antihypertensive treatment. 21 patients presented hypertension. Only 7 patients (2 patients of Group A, 4 patients of Group B and 1 patient of Group C) received anti-hypertensive treatment with thiazide diuretics, captopril/hydrochlorothiazide, metoprolol and losartan. Group A and Group B patients did not present microvascular diabetic complications such as proliferative diabetic retinopathy, microalbuminuria and polyneuropathy but some of them had CHD (Table 1) and were used as examples for macrovascular diabetic complications. All diabetic patients (Group A and $\mathrm{B}$ ) received oral hypoglycemic agents.

Statistical analysis of the data was performed using SPSS for Windows version 10 statistical software package [11]. Initially data were assessed through simple cross-tabulations and by using chi-square test, $t$-test for normally distributed variables, Wilcoxon test for not normally distributed variables, and one-way ANOVA test or Kruskal-Wallis test $[11,12]$. Normality hypothesis was tested by Kolmogorov-Smirnov test, measures of asymmetry and Shapiro-Wilk test. The Pearson or Spearman correlation coefficient ( $r$ ) was used as a measurement of correlation. Subsequently, statistical analysis was undertaken through multiple linear regression models $[11,12]$. For all tests performed, a two-sided pvalue of less than 0.05 was considered as significant.

\section{Results}

Table 1 depicts the demographic and clinical characteristics of patients and controls as well as the histologic subtype of MDS patients according to the FAB classification scheme. The mean age of diabetic MDS patients was 72.6 years $( \pm 6.1)$, of diabetic patients 70.2 years $( \pm 6.1)$ and of hospital controls 73.5 years $( \pm 5.9)$. Male patients in each group were $21(70 \%)$ and female $9(30 \%)$. The predominant histologic subtype of MDS was RA (56.7\%) with about more than half of all MDS cases diagnosed in this category.

Mean platelet count in diabetic MDS patients (Group A) was significantly lower than that in diabetic patients 
$(p<0.001)$ and controls $(p<0.001)$, but platelet count in all groups was within normal ranges $(150-400 \times$ $\left.10^{9} / \mathrm{L}\right)$. MPV and PDW in diabetic patients were significantly higher than those in diabetic MDS patients $(p<$ $0.001)$ and controls $(p<0.001)$.Fasting blood glucose and $\mathrm{HbA}_{1 c}$ in diabetic patients were significantly higher than those in diabetic MDS patients $(p=0.006$ and $p<0.001$ respectively) and controls ( $p<0.001)$. BMI in diabetic patients was significantly higher than BMI in diabetic MDS patients $(p<0.001)$ and controls $(p<$ $0.001)$.

In the group of diabetic MDS patients (Group A), there were no correlations between MPV and fasting glucose $(r=-0.157, p=0.408)$, and MPV and $\mathrm{HbA}_{1 c}$ $(r=0.014, p=0.941)$. In Group A, MPV correlated significantly with PDW $(r=0.853, p<0.001)$. MPV and PDW were statistically significantly higher in diabetic MDS patients with hypertension ( $p=0.03$ and 0.02 respectively) and in diabetic MDS patients with CHD ( $p=0.02$ and 0.05 respectively).

In the group of diabetic patients (Group B) there were statistically significant correlations between MPV and fasting glucose $(r=0.825, p<0.001)$, MPV and $\mathrm{HbA}_{1 c}(r=0.877, p<0.001)$, and MPV and PDW $(r=0.694, p<0.001)$. PDW correlated significantly with fasting serum glucose $(r=0.66 ; p<0.001)$ and $\mathrm{HbA}_{1 c}(r=0.579, p<0.001)$. MPV and PDW were statistically significantly higher in diabetic patients with hypertension $(p<0.001)$ and in diabetic patients with CHD ( $p=0.03$ and 0.006 respectively). Overall, in patients with diabetes (Group A and B), MPV and PDW were statistically significantly higher in patients with CHD ( $p=0.024$ and $p=0.036$ respectively) and hypertension $(p=0.05)$.

In controls (Group C), MPV correlated significantly with fasting glucose $(r=0.763, p<0.001)$ but not with $\mathrm{HbA}_{1 c}(r=-0.118, p=0.536)$. Similarly, PDW correlated significantly with fasting glucose $(r=0.439$, $p=0.015)$ but not with $\mathrm{HbA}_{1 c}(r=-0.024, p=0.9)$.

Multiple linear regression models were performed in order to explore the association between MPV and $\mathrm{HbA}_{1 c}$ /fasting blood glucose, adjusting for confounding factors such as age, gender, BMI, platelet count, CHD, hypertension in diabetic MDS patients, in diabetic patients and finally in controls (Tables 2, 3 and 4). Adjusting for age, gender, BMI, platelet number, CHD and hypertension, neither $\mathrm{HbA}_{1 c}$ nor fasting blood glucose presented any statistically significant association with MPV ( $p=0.55$ and $p=0.26$ respectively, Table $2 \mathrm{a}$ ) or PDW ( $p=0.66$ and $p=0.28$ respectively, Table 2b) in diabetic MDS patients. Only platelet
Table 2

a. Multiple linear regression analysis of MPV (as dependent variable) in MDS diabetic patients adjusting for age and gender (matched variables), $\mathrm{HbA}_{1 c}$ (model 1), fasting blood glucose (model 2), hypertension, CHD, BMI and platelet count (as independent variables); regression coefficients (b), standard error of $b$ $\left(\mathrm{SE}_{b}\right)$ and $\mathrm{t}$ statistic with corresponding $\mathrm{p}$-value. $\mathrm{HbA}_{1 c}$ and fasting blood glucose were not introduced simultaneously in a model because of co-linearity

\begin{tabular}{|c|c|c|c|c|}
\hline Independent variables & $\mathrm{b}$ & $\mathrm{SE}_{b}$ & t statistic & $\mathrm{p}$ value \\
\hline \multicolumn{5}{|l|}{ Model 1} \\
\hline $\mathrm{HbA}_{1 c}$ & 0.08 & 0.14 & 0.61 & 0.55 \\
\hline Hypertension & 0.32 & 0.35 & 0.89 & 0.38 \\
\hline CHD & 0.81 & 0.5 & 1.62 & 0.12 \\
\hline BMI & 0.06 & 0.15 & 0.4 & 0.69 \\
\hline Platelet number & 0.02 & 0.01 & 4.57 & $<0.001^{*}$ \\
\hline \multicolumn{5}{|l|}{ Model 2} \\
\hline Fasting blood glucose & -0.17 & 0.14 & -1.16 & 0.26 \\
\hline Hypertension & 0.33 & 0.34 & 0.97 & 0.34 \\
\hline CHD & 0.89 & 0.5 & 1.79 & 0.09 \\
\hline BMI & 0.1 & 0.14 & 0.77 & 0.45 \\
\hline Platelet number & 0.02 & 0.01 & 4.04 & $<0.001^{*}$ \\
\hline \multicolumn{5}{|c|}{$\begin{array}{l}\text { b. Multiple linear regression analysis of PDW (as dependent } \\
\text { variable) in MDS diabetic patients adjusting for age and gen- } \\
\text { der (matched variables), } \mathrm{HbA}_{1 c} \text { (model 1), fasting blood glucose } \\
\text { (model } 2 \text { ), hypertension, } \mathrm{CHD}, \mathrm{BMI} \text { and platelet count (as inde- } \\
\text { pendent variables); regression coefficients (b), standard error of } \\
\left.\text { b ( } \mathrm{SE}_{b}\right) \text { and t statistic with corresponding p-value }\end{array}$} \\
\hline Independent variables & $\mathrm{b}$ & $\mathrm{SE}_{b}$ & t statistic & $\mathrm{p}$ value \\
\hline \multicolumn{5}{|l|}{ Model 1} \\
\hline $\mathrm{HbA}_{1 c}$ & -0.06 & 0.14 & -0.44 & 0.66 \\
\hline Hypertension & 0.35 & 0.38 & 0.93 & 0.36 \\
\hline CHD & 0.57 & 0.53 & 1.08 & 0.29 \\
\hline BMI & 0.04 & 0.16 & 0.31 & 0.76 \\
\hline Platelet number & 0.01 & 0.006 & 2.24 & $0.03^{*}$ \\
\hline \multicolumn{5}{|l|}{ Model 2} \\
\hline Fasting blood glucose & -0.009 & 0.008 & -1.1 & 0.28 \\
\hline Hypertension & 0.28 & 0.36 & 0.77 & 0.45 \\
\hline CHD & 0.76 & 0.53 & 1.41 & 0.17 \\
\hline BMI & 0.02 & 0.15 & 0.17 & 0.87 \\
\hline Platelet number & 0.01 & 0.006 & 2.2 & $0.05^{*}$ \\
\hline
\end{tabular}

* statistically significant when $p \leqslant 0.05$.

count was significantly associated with MPV and PDW ( $p<0.001$ and $p=0.03$ respectively). Adjusting for age, gender, BMI, platelet number, CHD and hypertension, $\mathrm{HbA}_{1 c}$ and fasting blood glucose were the only statistically significant predictors of MPV $(p<0.001$, Table 3a) and PDW ( $p=0.014$ and 0.007 respectively, Table $3 b$ ) in diabetic patients. Finally, controlling for the aforementioned variables, fasting blood glucose, platelet count and the presence of hypertension were statistically significant predictors of MPV in healthy controls (Table 4a).

Further adjustment for hypertensive treatment in all models for all Groups (A, B and C) didn't alter the results. 
Table 3

a. Multiple linear regression analysis of MPV (as dependent variable) in diabetic patients adjusting for age and gender (matched variables), $\mathrm{HbA}_{1 c}$ (model 1), fasting blood glucose (model 2), hypertension, $\mathrm{CHD}, \mathrm{BMI}$ and platelet count (as independent variables); regression coefficients (b), standard error of $\mathrm{b}\left(\mathrm{SE}_{b}\right)$ and $\mathrm{t}$ statistic with corresponding p-value. $\mathrm{HbA}_{1 c}$ and fasting blood glucose were not introduced simultaneously in a model because of co-linearity

\begin{tabular}{lcccc}
\hline Independent variables & $\mathrm{b}$ & $\mathrm{SE}_{b}$ & $\mathrm{t}$ statistic & $\mathrm{p}$ value \\
\hline Model 1 & & & & \\
HbA $_{1 c}$ & 0.99 & 0.14 & 7.17 & $<0.001^{*}$ \\
Hypertension & 0.52 & 0.3 & 1.73 & 0.09 \\
CHD & 0.12 & 0.4 & 0.29 & 0.79 \\
BMI & 0.003 & 0.06 & 0.04 & 0.97 \\
Platelet number & -0.0007 & 0.003 & -0.24 & 0.81 \\
Model 2 & & & & \\
Fasting blood glucose & 0.87 & 0.16 & 5.26 & $<0.001^{*}$ \\
Hypertension & 0.23 & 0.34 & 0.57 & 0.57 \\
CHD & 0.01 & 0.49 & 0.02 & 0.98 \\
BMI & 0.04 & 0.08 & 0.48 & 0.63 \\
Platelet number & -0.003 & 0.003 & -1.04 & 0.31 \\
\hline
\end{tabular}

* statistically significant when $p \leqslant 0.05$.

b. Multiple linear regression analysis of PDW (as dependent variable) in diabetic patients adjusting for age and gender (matched variables), $\mathrm{HbA}_{1 c}$ (model 1), fasting blood glucose (model 2), hypertension, CHD, BMI and platelet count (as independent variables); regression coefficients (b), standard error of $b\left(\mathrm{SE}_{b}\right)$ and $\mathrm{t}$ statistic with corresponding p-value

\begin{tabular}{lllll}
\hline Independent variables & $\mathrm{b}$ & $\mathrm{SE}_{b}$ & $\mathrm{t}$ statistic & $\mathrm{p}$ value \\
\hline Model 1 & & & & \\
HbA $_{1 c}$ & 0.68 & 0.26 & 2.66 & $0.014^{*}$ \\
Hypertension & 1.09 & 0.55 & 2 & 0.06 \\
CHD & 0.39 & 0.65 & 0.61 & 0.55 \\
BMI & 0.19 & 0.13 & 1.49 & 0.15 \\
Platelet number & 0.0007 & 0.005 & -0.14 & 0.89 \\
Model 2 & & & & \\
Fasting blood glucose & 0.04 & 0.01 & 2.93 & $0.007^{*}$ \\
Hypertension & 0.7 & 0.58 & 1.19 & 0.24 \\
CHD & 0.39 & 0.63 & 0.62 & 0.54 \\
BMI & 0.24 & 0.12 & 1.89 & 0.07 \\
Platelet number & 0.0002 & 0.005 & 0.05 & 0.96 \\
\hline
\end{tabular}

${ }^{*}$ statistically significant when $p \leqslant 0.05$.

\section{Discussion}

Diabetes mellitus constitutes a complex disease which is characterized by chronic hyperglycemia, metabolic abnormalities and long-term macrovascular and microvascular abnormalities involving the blood vessels, eyes, kidneys and nerves. Platelet parameters such as high platelet counts and mainly high MPV have been reported in diabetic patients and they are likely to be associated with an increased risk of vascular disease $[1,13]$. Higher MPV in diabetic patients indicates larger platelet size suggesting stimulated thrombopoiesis and augmented platelet activation [3,13]. Platelet hyperactivity is accompanied by an increased production of thromboxane A 2 and/or a decreased syn-

\section{Table 4}

a. Multiple linear regression analysis of MPV (as dependent variable) in healthy controls adjusting for age and gender (matched variables), $\mathrm{HbA}_{1 c}$ (model 1), fasting blood glucose (model 2), hypertension, CHD, BMI and platelet count (as independent variables); regression coefficients (b), standard error of $\mathrm{b}\left(\mathrm{SE}_{b}\right)$ and $\mathrm{t}$ statistic with corresponding p-value. $\mathrm{HbA}_{1 c}$ and fasting blood glucose were not introduced simultaneously in a model because of co-linearity

\begin{tabular}{lrrrl}
\hline Independent variables & $\mathrm{b}$ & $\mathrm{SE}_{b}$ & $\mathrm{t}$ statistic & $\mathrm{p}$ value \\
\hline Model 1 & & & & \\
HbA $_{1 c}$ & -0.35 & 0.37 & -0.96 & 0.35 \\
Hypertension & 2.59 & 0.43 & 6.06 & $0.001^{*}$ \\
CHD & 0.14 & 0.91 & 0.15 & 0.88 \\
BMI & -0.03 & 0.11 & -0.27 & 0.79 \\
Platelet number & -0.01 & 0.003 & -3.66 & $0.001^{*}$ \\
Model 2 & & & & \\
Fasting blood glucose & 0.99 & 0.2 & 4.81 & $0.001^{*}$ \\
Hypertension & 2.05 & 0.31 & 6.45 & $0.001^{*}$ \\
CHD & -0.18 & 0.64 & -0.29 & 0.77 \\
BMI & -0.07 & 0.08 & -0.87 & 0.39 \\
Platelet number & -0.007 & 0.002 & -3.69 & $0.001^{*}$ \\
\hline
\end{tabular}

${ }^{*}$ statistically significant when $p \leqslant 0.05$.

b. Multiple linear regression analysis of PDW (as dependent variable) in healthy controls adjusting for age and gender (matched variables), $\mathrm{HbA}_{1 c}$ (model 1), fasting blood glucose (model 2), hypertension, CHD, BMI and platelet count (as independent variables); regression coefficients (b), standard error of $\mathrm{b}\left(\mathrm{SE}_{b}\right)$ and $\mathrm{t}$ statistic with corresponding p-value

\begin{tabular}{lccrc}
\hline Independent variables & $\mathrm{b}$ & $\mathrm{SE}_{b}$ & $\mathrm{t}$ statistic & $\mathrm{p}$ value \\
\hline Model 1 & & & & \\
HbA $_{1 c}$ & -0.36 & 0.52 & -0.69 & 0.49 \\
Hypertension & 3.51 & 0.56 & 6.23 & $<0.001^{*}$ \\
CHD & 0.93 & 1.11 & 0.84 & 0.41 \\
BMI & 0.13 & 0.16 & 0.81 & 0.43 \\
Platelet number & -0.009 & 0.004 & -2.43 & $0.02^{*}$ \\
Model 2 & & & & \\
Fasting blood glucose & -0.008 & 0.02 & -0.37 & 0.72 \\
Hypertension & 3.54 & 0.59 & 5.92 & $<0.001^{*}$ \\
CHD & 0.71 & 1.06 & 0.67 & 0.51 \\
BMI & 0.12 & 0.16 & 0.79 & 0.44 \\
Platelet number & -0.01 & 0.004 & -2.89 & $0.008^{*}$ \\
\hline
\end{tabular}

*statistically significant when $p \leqslant 0.05$.

thesis of prostacycline as larger platelets produce more serotonine, thromboxane A2, $\beta$-thromboglobulin and secrete more membrane receptors such as CD63 and CD62 [14,15].

In agreement with other studies [1,5], our study showed that diabetic patients presented significantly higher MPV and PDW than age- and gender-matched non-diabetic controls. Furthermore, both in univariate analysis and after adjustment for confounders, we found that there was a positive correlation between MPV and $\mathrm{HbA}_{1 c}$ or fasting blood glucose in diabetic patients. One possible mechanism of increased MPV in DM is osmotic swelling due to raised blood glucose and some glucose metabolites [16] and perhaps due to a shorter life span of platelets in diabetic patients [17]. 
Alternatively, this may also suggest that platelet activation is related to glycemic control, an issue of current debate $[1,5,18,19]$.

In contrast, we found no correlation between platelet parameters and $\mathrm{HbA}_{1 c}$ or fasting blood glucose in diabetic MDS patients with normal platelet count after adjusting for age, gender, BMI, platelet count, presence of CHD and hypertension. To the best of our knowledge, this is the first study in medical literature evaluating the association of glycemic control and platelet parameters in diabetic MDS patients with normal platelet count.

MDS constitutes a heterogeneous group of acquired clonal disorders of the stem cell, characterized by ineffective hematopoiesis leading to one or more peripheral blood cytopenias associated typically with a normocellular or hypercellular bone marrow [20]. Thrombocytopenia in MDS $\left(<100 \times 10^{9} / \mathrm{L}\right)$ ranges from $40 \%$ to $65 \%$ [21]. Recently, it has been reported that MPV is lower in thrombocytopenic patients with bone marrow disease and could be used as a surrogate prognostic marker for the degree of bone marrow disease in MDS patients [8]. In our study, all MDS patients had normal platelet count (mean: $177.9 \times 10^{9} / \mathrm{L}$; range: 150 $290 \times 10^{9} / \mathrm{L}$ ) but presented lower MPV (mean: $9.5 \mathrm{fL}$; range: $8-12 \mathrm{fL}$ ) than diabetics and controls. Low MPV in myelodysplastic patients with normal platelet count may represent a disease marker for bone marrow dysplasia as well as a prognostic marker for MDS status [8]. Based upon the non-associations of $\mathrm{HbA}_{1 c}$ as well as fasting blood glucose with MPV in this group of patients, it could be inferred that indices of glycemic control didn't contribute to platelet morphology and that other factors inherent to bone marrow dysplasia pathogenesis, platelet turnover, platelet biochemistry, or vascular environment could be more important determinants of platelet size and function. Platelet dysfunction and especially hypoactivity has been reported in MDS patients with platelet count greater than $80 \times$ $10^{9} / \mathrm{L}$ [22]. Moreover, independently of the category of diabetic patients (MDS patients versus DM patients), CHD (as an example of DM macrovascular complication) and hypertension were associated with increased MPV in univariate analysis only, in agreement with studies reporting that MPV is associated with increased cardiovascular morbidity [7] and hypertension [23].

In non-diabetic, non-MDS controls, fasting blood glucose, platelet number and the presence of hypertension predicted significantly platelet morphology represented by MPV. This finding is consistent with a similar study reporting that mean platelet volume is significantly higher in hypertensive patients indicating the impor- tance of platelet morphology and function as emerging risk factors for atherothrombosis [23].

The main limitation of our study is the relatively small sample size which is due to the rare incidence of MDS patients with co-existing DM. Nevertheless, we are able to observe statistically significant associations between glycemic control indices and platelet morphologic parameters in diabetics with or without MDS, and to show these groups respond differently. The inclusion of a non-diabetic MDS control group would be necessary to further explore the relationship.

In conclusion, higher MPV and higher PDW are associated with higher mean glycosylated hemoglobin and higher fasting glucose in diabetic, but not matched diabetic myelodysplastic patients presenting with normal platelet counts. This may reflect MPV as a disease marker for diabetes control and thrombotic risk in diabetics. Moreover, it points to non-glycemic factors in the diabetic MDS patient including undetected bone marrow dysplasia, platelet biochemical variations, platelet turnover rates, or vascular changes. Further study is needed to assess the association of platelet morphology with macrovascular disease or risk; to determine the impact of risk reducing interventions; and to elucidate these findings in the setting of bone marrow dysfunction.

\section{Acknowledgement}

Hellenic Harvard Foundation provided support to Dr Maria Dalamaga.

\section{Conflict of interest statement}

There is no any conflict of interest

\section{References}

[1] N. Papanas, G. Symeonidis, E. Maltezos, G. Mavridis, F. Karavageli, T. Vosnakidis and G. Lakasas, Mean platelet volume in patients with type 2 diabetes mellitus, Platelets 15 (2004), 475-478.

[2] P.M.W. Bath, C.G. Missouris, T. Buckenham and G.A. McGregor, Increased platelet volume and platelet mass in patients with atherosclerotic renal artery stenosis, Clin Sci 87 (1994), 253-257.

[3] P.M.W. Bath and R.J. Butterworth, Platelet size: measurement, physiology and vascular disease, Blood Coagul Fibrinolysis 7 (996), 431-436. 
[4] J.F. Martin, E.A. Trowbridge, G. Salmon and J. Plumb, The biological significance of platelet volume: its relationship to bleeding time, platelet thromboxane A2 production and megakaryocyte nuclear DNA concentration, Thromb Res 32 (1983), 443-460.

[5] P.C. Sharpe and T. Trinick, Mean platelet volume in diabetes mellitus, Q J Med 86, (1993), 739-742.

[6] L. Mazzanti and B. Mutus, Diabetes-induced alterations in platelet metabolism, Clin Biochem 30 (1997), 509-515.

[7] N. Kilicli-Camur, R. Demirtunc, C. Konuralp, A. Eskiser and Y. Basaran, Could mean platelet volume be a predictive marker for acute myocardial infarction? Med Sci Monit 11 (2005), 387-392.

[8] K.M. Bowles, B.A. Warner and T.P. Baglin, Platelet mass has prognostic value in patients with myelodysplastic syndromes, Br J Haematol 135 (2006), 198-200.

[9] M. Bennett, D. Catovsky, M.T. Daniel, G. Flandrin, D.A. Galton, H.R. Gralnick and C. Sultan. Proposals for the classification of MDS, Br J Haematol 51 (1982), 189.

[10] American Diabetes Association: diagnosis and classification of diabetes mellitus, Diabetes Care 27(1) (2004), 5-10.

[11] SPSS ${ }^{\circledR}$ Base 10.0 Applications Guide. Chicago, IL: SPSS Inc., 1989.

[12] B.C. Cronk, How to use SPSS ${ }^{\circledR}$, A step-by-step guide to analysis and interpretation, Pyrczak Publishing, Los Angeles, 1999, pp. 39-50.

[13] D. Tschoepe, P. Roesen, B. Schwippert and F.A. Gries, Platelets in Diabetes: the role in the hemostatic regulation in atherosclerosis, Semin Thromb Haemost 9 (1993), 122-128.

[14] D. Tschoepe, P. Roesen, J. Esser, B. Schwippert, H.K. Nieuwenhuis, B. Kehrel and F.A. Gries, Large platelets circulate in an activated state in diabetes mellitus, Semin Thromb Haemost 17 (1991), 433-438.
[15] S. Srivastava, C.S. Joshi, P.P. Sethi, A.K. Agrawal, S.K. Srivastava and P.K. Seth, Altered platelet functions in non-insulindependent diabetes mellitus (NIDDM), Thromb Res 76 (1994), 451-461.

[16] C.N. Martyn, D.M. Matthews, C. Popp-Snijders, J. Tucker, D.J. Ewing and B.F. Clarke, Effects of sorbinil treatment on erythrocytes and platelets of persons with diabetes, Diabetes Care 9 (1986), 36-39.

[17] R.L. Jones, C. Paradise and C.M. Peterson, Platelet survival in patients with diabetes mellitus, Diabetes 30 (1981), 486-489.

[18] Y. Inui, T. Suehiro, Y. Kumon and K. Hashimoto, Platelet volume and urinary prostanoid metabolites in non-insulindependent diabetes mellitus, J Atheroscler Thromb 1 (1994), 108-112.

[19] E. Coban, F. Bostan and M. Ozdogan, The mean platelet volume in subjects with impaired fasting glucose, Platelets 17 (2006), 67-69.

[20] A.F. List and D.F. Doll, The Myelodysplastic Syndromes, in: Wintrobe's Clinical Hematology, G.R. Lee, J. Foerster, J. Lukens, F. Paraskevas, J.P. Greer and G.M. Rodgers, eds, 10th edition, Williams and Wilkins, Baltimore, 1999, pp. 23202333.

[21] H. Kantarjian, F. Giles, A. List, R. Lyons, M.A. Sekeres, S. Pierce, R. Deuson and J. Leveque, The incidence and impact of thrombocytopenia in myelodysplastic syndromes, Cancer 109 (2007), 1705-1714.

[22] A. Manoharan, T. Brighton, G.K. Lopez, S. Moran and P. Kyle, Platelet dysfunction in Myelodysplastic Syndromes: a clinicopathological study, Int J Hematol 76 (2002), 272-278.

[23] E. Coban, G. Yazicioglu, A. Berkant Avci and F. Akcit, The mean platelet volume in patients with essential and white coat hypertension, Platelets 16 (2005), 435-438. 


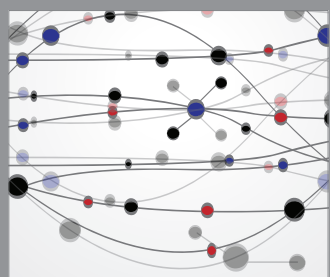

The Scientific World Journal
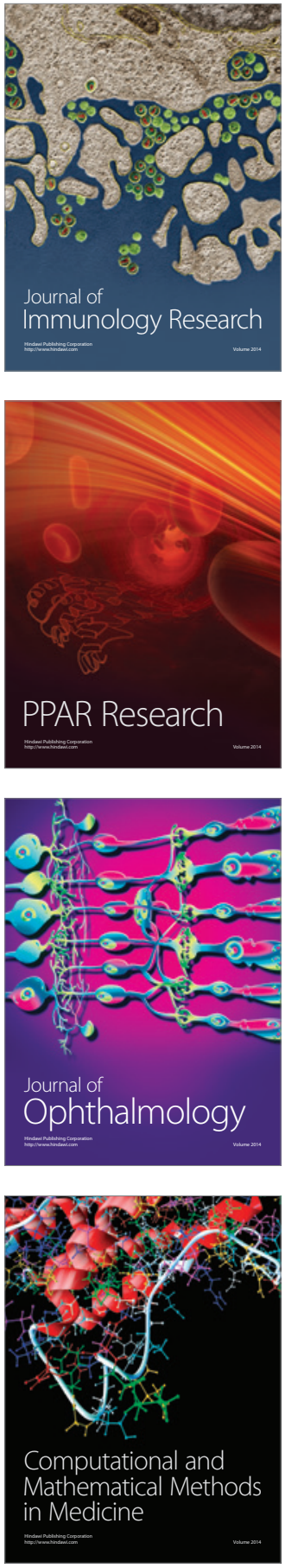

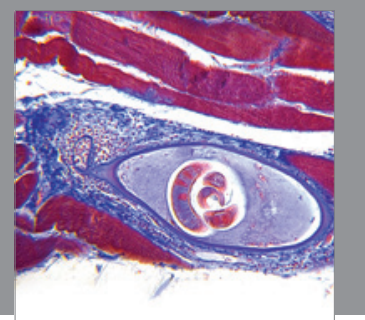

Gastroenterology

Research and Practice
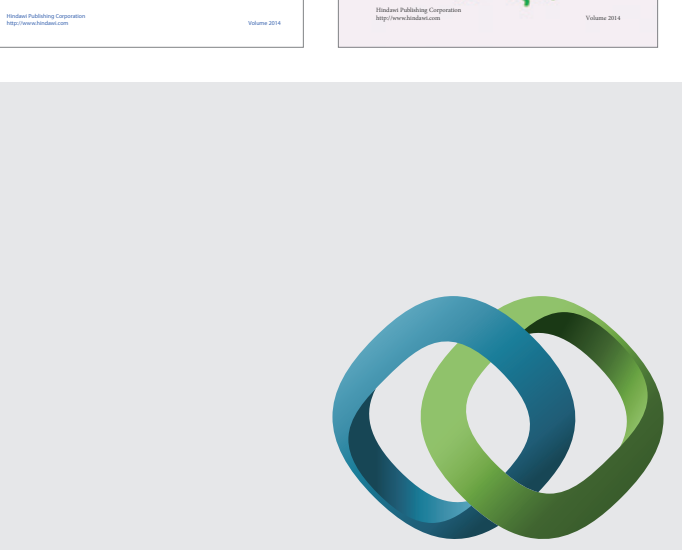

\section{Hindawi}

Submit your manuscripts at

http://www.hindawi.com
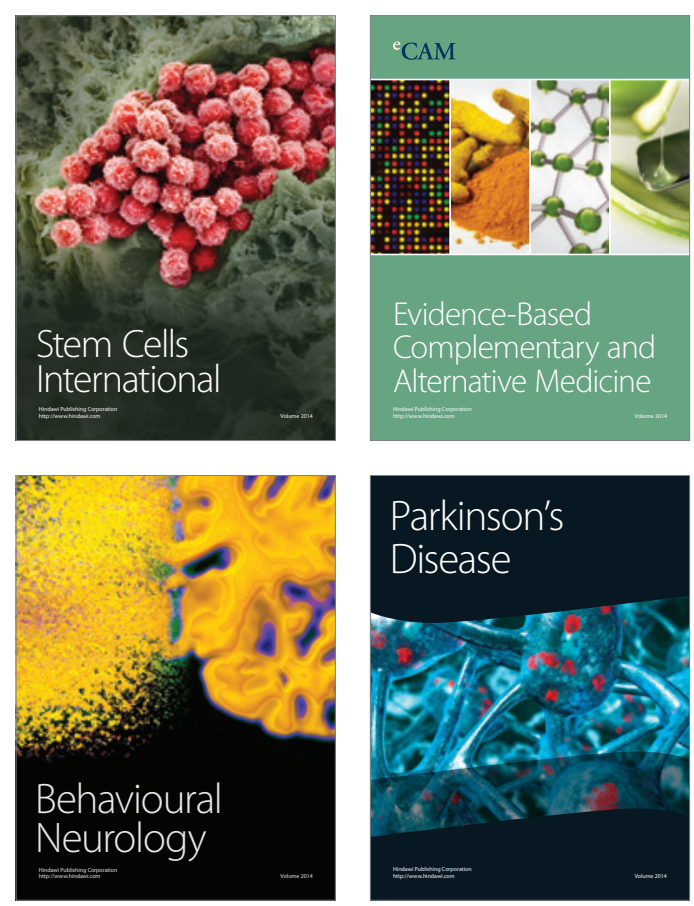

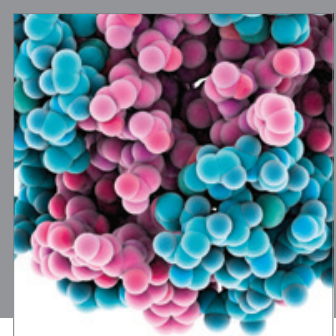

Journal of
Diabetes Research

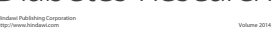

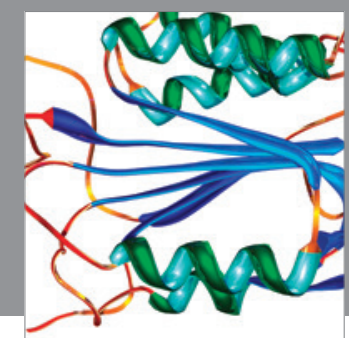

Disease Markers
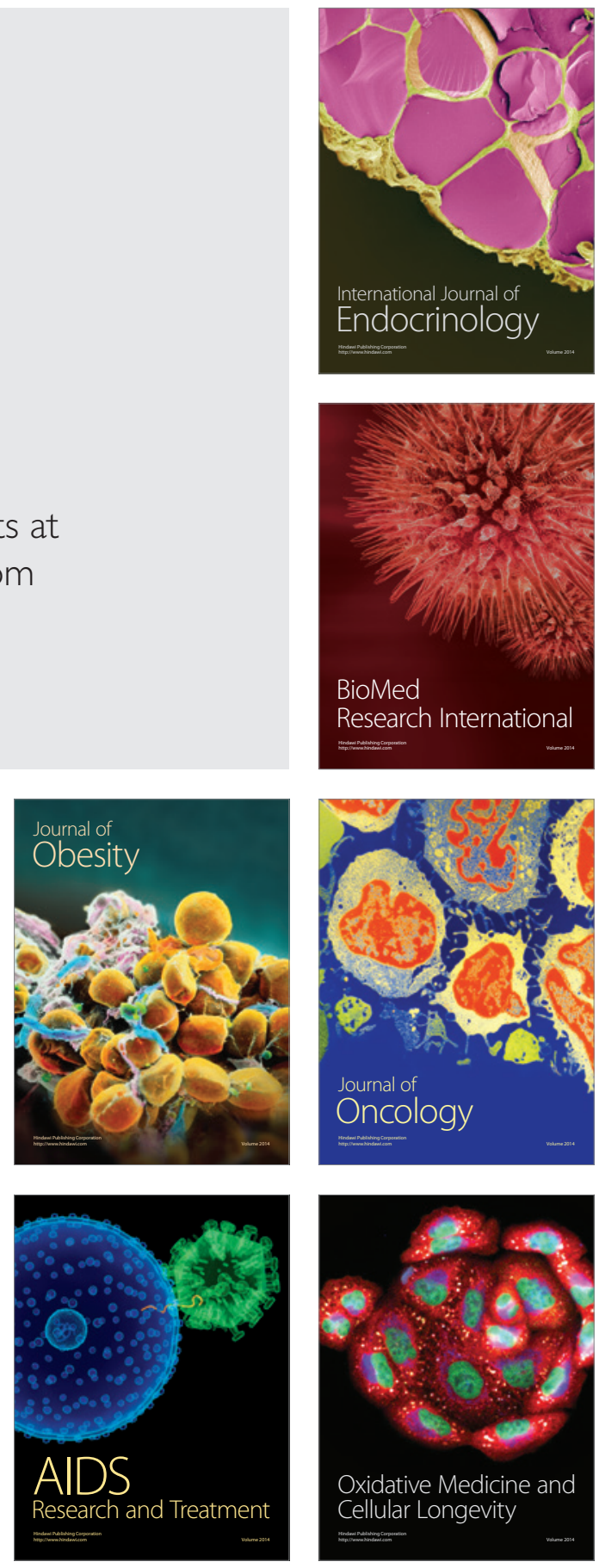